\title{
The Positive Role of Multiplicative Noise in Complete Synchronization of Unidirectionally Coupled Ring with Three Nodes
}

\author{
Yuzhu Xiao, ${ }^{1}$ Sufang Tang, ${ }^{2}$ and Zhongkui Sun ${ }^{3}$ \\ ${ }^{1}$ Department of Mathematics and Information Science, Chang'an University, Xian 710064, China \\ ${ }^{2}$ School of Statistics, Xian University of Finance and Economics, Xian 710100, China \\ ${ }^{3}$ Department of Applied Mathematics, Northwestern Polytechnical University, Xian 710072, China \\ Correspondence should be addressed to Yuzhu Xiao; yuzhuxiao@chd.edu.cn
}

Received 28 January 2014; Revised 21 June 2014; Accepted 22 June 2014; Published 16 July 2014

Academic Editor: Yongkun Li

Copyright (C) 2014 Yuzhu Xiao et al. This is an open access article distributed under the Creative Commons Attribution License, which permits unrestricted use, distribution, and reproduction in any medium, provided the original work is properly cited.

\begin{abstract}
The role of multiplicative noise in the synchronization of unidirectionally coupled ring with three nodes is studied. Based on the theory of stochastic differential equations, we demonstrate that noise plays a positive role in complete synchronization. In numerical simulations, the Lorenz system, Rössler like system, and Hindmarsh-Rose neuron model are employed to demonstrate the correctness of our theoretical result.
\end{abstract}

\section{Introduction}

Synchronization is an omnipresent phenomenon in nonlinear systems wherein two or more systems coupled by a suitable configuration or driven by external forcing adjust one property of their motion to a common behavior $[1,2]$. With the development of chaos synchronization, different kinds of synchronization have been found, such as complete synchronization [2-5], phase synchronization [6], lag synchronization $[7,8]$, and generalized synchronization $[9,10]$. The synchronization of nonlinear systems has many potential applications in different areas, such as secure communication $[11,12]$, biological systems [13, 14], chemical reactions [15], and physical systems [16]. Due to the potential applications of synchronization, some synchronization methods have been proposed, such as active control [17], adaptive control [18], sliding control [19], and integral observer [20].

Since noise is ubiquitous in real-world systems, the synchronization of coupled systems is unavoidably affected by different kinds of noise. Therefore, the role of noise in synchronization is very important and has been extensively studied. Intuitively, noise is random disturbance, which should play a destroyed role in the synchronization of coupled systems. However, some recent studies have shown that synchronization can be induced by noise. Firstly, noise-induced synchronization was found in periodic systems [21, 22]. With the development of chaos synchronization, the phenomenon of noise-induced synchronization was extended to chaotic map [23]. The result in [23] has spurred a long-standing dispute since it was found. Firstly, Pikovsky pointed out that the result in [23] is not right, because the largest Lyapunov exponent is positive [24]. Some other authors argued that synchronization in [23] is caused by biased noise. One explanation is that biased noise can move the system into "convergence region," so unbiased noise can not induce synchronization [25-27]. However, some recent work showed that some chaotic systems can be synchronized by unbiased additive noise $[28,29]$. With the extensive study of noise's effect on synchronization, the phenomenon of noiseinduced synchronization has been extended to generalized synchronization $[30,31]$, phase synchronization $[32,33]$, and lag synchronization [34]. However, the above studies mostly concentrate on the case of additive noise, and most of results are based on numerical simulations and experiments.

Recently, the study of noise's effect on synchronization is extended to multiplicative noise. In [35], the authors 
studied two coupled chaotic systems perturbed by multiplicative noise. Based on the invariance principle of stochastic differential equations, the sufficient condition ensuring the complete synchronization was established. Sooner, above method was generalized to coupled chaotic systems with multiple time delays [36] and linear generalized synchronization [37]. In [38], the sufficient conditions of complete synchronization of linear stochastic coupled network under adaptive control were established. However, the role of noise in synchronization was not discussed. In [39], the positive role of multiplicative noise in synchronization was revealed in two unidirectionally coupled chaotic systems, and authors proposed a good analytical method to analyze this phenomenon. In [40], two bidirectionally coupled chaotic systems were studied by above method. In this paper, the method in [39] will be extended to three coupled chaotic systems with unidirectional ring structure. The coupled ring has been used widely in modeling physiological, biochemical, and biological phenomena $[13,14]$. Though many real-world systems should be described by the network with many nodes and complex structure, the ring with three nodes is the network motifs of some complex networks. The investigation of the synchronization of ring with three nodes is helpful to understand the collective behavior in complex networks [41].

\section{Theoretical Analysis}

The unidirectionally coupled ring with three nodes can be described as follows:

$$
\begin{aligned}
& \dot{\mathbf{x}}_{1}=\mathbf{f}\left(\mathbf{x}_{1}\right)+c\left(\mathbf{x}_{2}-\mathbf{x}_{1}\right), \\
& \dot{\mathbf{x}}_{2}=\mathbf{f}\left(\mathbf{x}_{2}\right)+c\left(\mathbf{x}_{3}-\mathbf{x}_{2}\right), \\
& \dot{\mathbf{x}}_{3}=\mathbf{f}\left(\mathbf{x}_{3}\right)+c\left(\mathbf{x}_{1}-\mathbf{x}_{3}\right) .
\end{aligned}
$$

Here $\mathbf{x}_{i}=\left(x_{i 1}, x_{i 2}, \ldots, x_{i m}\right)^{T} \in R^{m}$ is the state vector of node $i(i=1,2,3)$, and $\mathbf{f}=\left(f_{1}, f_{2}, \ldots, f_{m}\right)^{T}$ is the vector function which describes the dynamic of uncoupled system. $c$ is a positive constant, which is usually called coupling strength. Consider that the real-world systems are unavoidably disturbed by internal noise which may lead to the loss of information in transmission process [38]; then the coupled ring model (1) can be improved as follows:

$$
\begin{aligned}
& \dot{\mathbf{x}}_{1}=\mathbf{f}\left(\mathbf{x}_{1}\right)+(c+d \xi(t))\left(\mathbf{x}_{2}-\mathbf{x}_{1}\right), \\
& \dot{\mathbf{x}}_{2}=\mathbf{f}\left(\mathbf{x}_{2}\right)+(c+d \xi(t))\left(\mathbf{x}_{3}-\mathbf{x}_{2}\right), \\
& \dot{\mathbf{x}}_{3}=\mathbf{f}\left(\mathbf{x}_{3}\right)+(c+d \xi(t))\left(\mathbf{x}_{1}-\mathbf{x}_{3}\right),
\end{aligned}
$$

where $d$ is a constant which describes the noise intensity, and $\xi(t)$ is one-dimensional Gaussian white noise with statistical properties $\langle\xi(t)\rangle=0$ and $\left\langle\xi(t), \xi\left(t^{\prime}\right)\right\rangle=\delta\left(t-t^{\prime}\right)$. In fact, the assumption of Gaussian white noise is often impractical, but this assumption is a good approximation of many real-world situations and generates mathematically tractable models. The noise term in (2) is of multiplicative form, so (2) can be interpreted in the sense of Itô or Stratonovich. The Itô sense is employed here; though this interpretation seems to be mathematical limiting procedure, this is reasonable when the rapidity of fluctuation of environment is far less than the macroscopic time scale of the system, such as the external fluctuation in many biological systems [39, 42]. In most of real physical systems, the Stratonovich sense is usually employed. However, the recent study shows that the multiplicative noise in electric circuit, which usually obeys the Stratonovich convention, crosses over to obey the Itô convention when the certain parameters of the systems are changed [43]. In a word, the Itô interpretation of model is not totally idealizing but is realizable in many real-world systems.

To begin the theoretical analysis, the coupled ring (2) and the vector function $\mathbf{f}=\left(f_{1}, f_{2}, \ldots, f_{m}\right)^{T}$ need to satisfy the following assumption.

Assumption. Assume that the coupled ring (2) is bounded, and for any bounded set $\Omega \in R^{m}$, there exists a positive constant $l$ such that $\mathbf{x}, \mathbf{y} \in \Omega$ satisfies

$$
(\mathbf{x}-\mathbf{y})^{T}[f(\mathbf{x})-f(\mathbf{y})] \leq l(\mathbf{x}-\mathbf{y})^{T}(\mathbf{x}-\mathbf{y}),
$$

where $l$ is a positive constant. Notice that this condition is very loose; for example, the condition (3) is satisfied as long as $\partial f_{i} / \partial x_{i}$ are bounded on $\Omega$. Therefore, most of well-known chaotic systems satisfy condition (3).

Actually, the coupled ring (2) is said to be synchronized if $\mathbf{x}_{1}=\mathbf{x}_{2}=\mathbf{x}_{3} \rightarrow \mathbf{s}(t)$ as $t \rightarrow \infty$. $\mathbf{s}(t) \in R^{m}$ is called synchronized manifold which is usually a solution of uncoupled system; that is, $\dot{\mathbf{s}}=\mathbf{f}(\mathbf{s})$. Inspired by [44], the manifold $\overline{\mathbf{s}}(t)=\left(\mathbf{x}_{1}+\mathbf{x}_{2}+\mathbf{x}_{3}\right) / 3$ is introduced to study the stability of $\mathbf{s}(t)$. This manifold has some new properties which is helpful to theoretical analysis. It is easy to see that

$$
\dot{\overline{\mathbf{s}}}(t)=\frac{1}{3} \sum_{i=1}^{3} \mathbf{f}\left(\mathbf{x}_{i}\right) \triangleq \mathbf{g}(\overline{\mathbf{s}}) .
$$

Define $\mathbf{e}_{i}=\mathbf{x}_{i}-\overline{\mathbf{s}}(t)$ to be the error state between the state of $i$ th node and manifold $\overline{\mathbf{s}}(t)$. Obviously, the error states are dominated by the following equation:

$$
\begin{aligned}
& \dot{\mathbf{e}}_{1}=\mathbf{f}\left(\mathbf{x}_{1}\right)-\mathbf{g}(\overline{\mathbf{s}}(t))+(c+d \xi(t))\left(-\mathbf{e}_{1}+\mathbf{e}_{2}\right), \\
& \dot{\mathbf{e}}_{2}=\mathbf{f}\left(\mathbf{x}_{2}\right)-\mathbf{g}(\overline{\mathbf{s}}(t))+(c+d \xi(t))\left(-\mathbf{e}_{2}+\mathbf{e}_{3}\right), \\
& \dot{\mathbf{e}}_{3}=\mathbf{f}\left(\mathbf{x}_{3}\right)-\mathbf{g}(\overline{\mathbf{s}}(t))+(c+d \xi(t))\left(-\mathbf{e}_{3}+\mathbf{e}_{1}\right) .
\end{aligned}
$$

Here, one should notice that error states $\mathbf{e}_{i}(i=1,2,3)$ satisfy the following equality:

$$
\mathbf{e}_{1}+\mathbf{e}_{2}+\mathbf{e}_{3}=0
$$

This property is very useful in the following theoretical analysis.

For convenience of expression, (5) is rewritten as the following matrix form:

$$
\dot{\mathbf{E}}(t)=\mathbf{F}(\mathbf{x})+\mathbf{C}(\mathbf{E})+\mathbf{H}(\mathbf{E}) \dot{\mathbf{W}}(t) .
$$


Here

$$
\begin{gathered}
\mathbf{E}(t)=\left(\begin{array}{l}
\mathbf{e}_{1} \\
\mathbf{e}_{2} \\
\mathbf{e}_{3}
\end{array}\right), \quad \mathbf{F}(\mathbf{x})=\left(\begin{array}{l}
\mathbf{f}\left(\mathbf{x}_{1}\right)-\mathbf{g}(\mathbf{s}(t)) \\
\mathbf{f}\left(\mathbf{x}_{2}\right)-\mathbf{g}(\mathbf{s}(t)) \\
\mathbf{f}\left(\mathbf{x}_{2}\right)-\mathbf{g}(\mathbf{s}(t))
\end{array}\right), \\
\mathbf{C}(\mathbf{E})=c\left(\begin{array}{c}
-\mathbf{e}_{1}+\mathbf{e}_{2} \\
-\mathbf{e}_{2}+\mathbf{e}_{3} \\
-\mathbf{e}_{3}+\mathbf{e}_{1}
\end{array}\right), \quad \mathbf{H}(\mathbf{E})=d\left(\begin{array}{l}
-\mathbf{e}_{1}+\mathbf{e}_{2} \\
-\mathbf{e}_{2}+\mathbf{e}_{3} \\
-\mathbf{e}_{3}+\mathbf{e}_{1}
\end{array}\right), \\
\dot{W}(t)=\xi(t) .
\end{gathered}
$$

According to the stability theory of stochastic differential equations $[39,45]$, the error equation (7) possesses a unique global solution with any initial state, without loss of generality, denoted by $\mathbf{E}\left(t ; t_{0}, \mathbf{E}_{0}\right)$. It is easy to see that $\mathbf{E}(t ; 0, \mathbf{0}) \equiv \mathbf{0}$ is a trivial solution of the error system (7). The complete synchronization of dynamical network (2) is achieved, if the zero solution is asymptotically stable; that is, $\lim _{t \rightarrow \infty}\|\mathbf{E}(t)\|=$ 0 with probability one.

In the following, the role of noise in the synchronization of the coupled ring (2) will be analyzed by the theory of stochastic differential equations. To this end, the following function is defined:

$$
V(\mathbf{E})=\frac{1}{2} \log \left(\mathbf{E}^{T}(t) \mathbf{E}(t)\right)
$$

By [39], $\mathbf{E}\left(t ; t_{0}, \mathbf{E}_{0}\right)$ never reaches zero with probability one if $\mathbf{E}_{0} \neq 0$. This ensures the correctness of adopting the logarithm in (9). By applying Itô formula to (9) along with system (7) $[39,45]$, one has

$$
\begin{aligned}
& V(\mathbf{E}(t)) \\
& =V\left(\mathbf{E}\left(t_{0}\right)\right) \\
& \quad+\int_{t_{0}}^{t}\{D V(\mathbf{E}(s))[\mathbf{F}(\mathbf{x})+\mathbf{C}(\mathbf{E})] \\
& \left.\quad+\frac{1}{2} \operatorname{trace}\left[\mathbf{H}^{T}(\mathbf{E}(s)) D^{2}(V(\mathbf{E}(s))) \mathbf{H}(\mathbf{E}(s))\right]\right\} d s \\
& \quad+M(t),
\end{aligned}
$$

where

$$
\begin{gathered}
D V(\mathbf{E})=\frac{\mathbf{E}^{T}(t)}{\mathbf{E}^{T}(t) \mathbf{E}(t)}, \\
D^{2} V(\mathbf{E})=\frac{I}{\mathbf{E}^{T}(t) \mathbf{E}(t)}-2 \frac{\mathbf{E}(t) \mathbf{E}^{T}(t)}{\left(\mathbf{E}^{T}(t) \mathbf{E}(t)\right)^{2}},
\end{gathered}
$$

and the continuous martingale is

$$
M(t)=\int_{t_{0}}^{t} \frac{\mathbf{E}^{T}(s) \mathbf{H}(\mathbf{E}(t))}{\mathbf{E}^{T}(s) \mathbf{E}(s)} d W(s)
$$

The quadratic variation of (12) is

$$
\begin{aligned}
& {[M(t), M(t)]} \\
& \quad=\int_{t_{0}}^{t} \frac{\left[\mathbf{E}^{T}(s) \mathbf{H}(\mathbf{E}(t))\right]^{2}}{\left[\mathbf{E}^{T}(s) \mathbf{E}(s)\right]^{2}} d s \\
& =d^{2} \int_{t_{0}}^{t} \frac{\left[-\sum_{i=1}^{3} \mathbf{e}_{i}^{T} \mathbf{e}_{i}+\mathbf{e}_{1}{ }^{T} \mathbf{e}_{2}+\mathbf{e}_{2}{ }^{T} \mathbf{e}_{3}+\mathbf{e}_{3}{ }^{T} \mathbf{e}_{1}\right]^{2}}{\left(\sum_{i=1}^{3} \mathbf{e}_{i}^{T} \mathbf{e}_{i}\right)^{2}} d s \\
& \quad=d^{2} \int_{t_{0}}^{t} \frac{\left[-(3 / 2) \sum_{i=1}^{3} \mathbf{e}_{i}^{T} \mathbf{e}_{i}\right]^{2}}{\left(\sum_{i=1}^{3} \mathbf{e}_{i}{ }^{T} \mathbf{e}_{i}\right)^{2}} d s=\frac{9}{4} d^{2}\left(t-t_{0}\right) .
\end{aligned}
$$

Notice that the condition (6) and the equality

$$
\mathbf{e}_{i}^{T} \mathbf{e}_{j}=\frac{1}{2}\left(\mathbf{e}_{i}+\mathbf{e}_{j}\right)^{T}\left(\mathbf{e}_{i}+\mathbf{e}_{j}\right)-\frac{1}{2}\left(\mathbf{e}_{i}^{T} \mathbf{e}_{i}+\mathbf{e}_{j}^{T} \mathbf{e}_{j}\right)
$$

are used in above proof.

By the strong law of large numbers $[39,45]$, one has that

$$
\lim _{t \rightarrow \infty} \frac{M(t)}{t}=0
$$

holds almost sure.

Substituting $\mathbf{E}(t), D V(\mathbf{E}), D^{2} V(\mathbf{E}), \mathbf{F}(\mathbf{x}), \mathbf{C}(\mathbf{E})$, and $\mathbf{H}(\mathbf{E})$ into (10) and applying equality (6), one has

$$
\begin{aligned}
V(\mathbf{E}(t)) & \\
= & V\left(\mathbf{E}\left(t_{0}\right)\right)+\int_{t_{0}}^{t} \frac{\sum_{i=1}^{3} \mathbf{e}_{i}^{T}\left[\mathbf{f}\left(\mathbf{x}_{i}\right)-\mathbf{f}(\mathbf{s}(t))\right]}{\sum_{i=1}^{3} \mathbf{e}_{i}^{T} \mathbf{e}_{i}} d s \\
& +c \int_{t_{0}}^{t} \frac{-(3 / 2) \sum_{i=1}^{3} \mathbf{e}_{i}^{T} \mathbf{e}_{i}}{\sum_{i=1}^{3} \mathbf{e}_{i}^{T} \mathbf{e}_{i}} d s \\
& +\frac{1}{2} d^{2} \int_{t_{0}}^{t}\left\{\frac{\sum_{i=1}^{3} 3 \mathbf{e}_{i}^{T} \mathbf{e}_{i}}{\sum_{i=1}^{3} \mathbf{e}_{i}^{T} \mathbf{e}_{i}}-2 \frac{\left[-(3 / 2) \sum_{i=1}^{3} \mathbf{e}_{i}^{T} \mathbf{e}_{i}\right]^{2}}{\left(\sum_{i=1}^{3} \mathbf{e}_{i}^{T} \mathbf{e}_{i}\right)^{2}}\right\} d s \\
& +M(t) \\
= & V\left(\mathbf{E}\left(t_{0}\right)\right)+\int_{t_{0}}^{t} \frac{\sum_{i=1}^{3} \mathbf{e}_{i}^{T}\left[\mathbf{f}\left(\mathbf{x}_{i}\right)-\mathbf{f}(\mathbf{s}(t))\right]}{\sum_{i=1}^{3} \mathbf{e}_{i}^{T} \mathbf{e}_{i}} d s \\
& -\frac{3}{2} c\left(t-t_{0}\right)-\frac{3}{4} d^{2}\left(t-t_{0}\right)+M(t) .
\end{aligned}
$$

If the coupled ring (2) is bounded and vector function $\mathbf{f}$ satisfies the condition (3), it yields

$$
\begin{aligned}
V(\mathbf{E}(t)) \leq & V\left(\mathbf{E}\left(t_{0}\right)\right)+l\left(t-t_{0}\right)-\frac{3}{2} c\left(t-t_{0}\right) \\
& -\frac{3}{4} d^{2}\left(t-t_{0}\right)+M(t) .
\end{aligned}
$$

Combining (15), one obtains

$$
\lim _{t \rightarrow \infty} \sup \frac{V(\mathbf{E}(t))}{t} \leq l-\frac{3}{2} c-\frac{3}{4} d^{2} .
$$


Obviously, if $l-(3 / 2) c-(3 / 4) d^{2}<0$, the logarithm function $V(\mathbf{E}(t))$ approaches to $-\infty$ as $t \rightarrow+\infty$; that is, $\mathbf{E}(t)$ approaches to zero as $t \rightarrow+\infty$. In other words, the trivial solution $\mathbf{E}(t ; 0, \mathbf{0}) \equiv \mathbf{0}$ of error system (7) is asymptotically stable with probability one when $l<(3 / 2) c+(3 / 4) d^{2}$. Moreover, the last term about the noise intensity in inequality (18) is negative, so the noise term has positive effect on the synchronization of the coupled ring (2). Particularly, when coupling strength $c=0$, the coupled ring (2) can synchronize with $l<(3 / 4) d^{2}$. This means synchronization may be achieved when all nodes are just coupled by multiplicative noise.

Remark 1. One should notice that the above inequality (18) is acquired with bounded assumption of coupled ring (2) and the condition (3). For large noise intensity, the coupled ring (2) may become unbounded. Though it is difficult for us to acquire the bounded condition of coupled ring (2), coupled ring (2) is usually bounded when noise intensity $d$ is small.

\section{Numerical Examples}

To demonstrate that noise term in coupled ring (2) plays a positive role in synchronization, the Lorenz systems [46], Hindmarsh-Rose neuron system [47], and Rössler like system [48] are employed to describe the dynamic of uncoupled system. In numerical simulations, the weak order 2 RungeKutta scheme with time step $\Delta t=10^{-2}$ is adopted to solve the stochastic differential equations [49].

To simplify the representation, a scale is defined to measure the synchronization of the coupled ring (2), which is

$$
\Delta(d)=\left\langle\frac{1}{3} \frac{\sum_{k=1}^{3} \sum_{j=1}^{m} \int_{T_{1}}^{T_{2}}\left|x_{k j}(t, \omega, d)-\bar{S}_{j}(t, \omega, d)\right| d t}{T_{2}-T_{1}}\right\rangle,
$$

where $\omega \in \Omega, \Omega$ denotes the set of all elementary events, $\langle\cdot\rangle$ represents sample average, and $m$ is the dimension of node system. According the definition, $\Delta(d)$ can be used to exhibit effect of synchronization with respect to noise intensity.

Example 1 (Lorenz system). The famous Lorenz system can be described as follows:

$$
\begin{aligned}
& \dot{x}=-\sigma(x-y), \\
& \dot{y}=-x z+r x-y, \\
& \dot{z}=x y-b z .
\end{aligned}
$$

Here $x, y$, and $z$ make up the system state, and $\sigma, r, b$ are positive system parameters. In this example, let $\sigma=10, r=$ $24.1, b=8 / 3$. Under this group of parameters, system (20) has chaotic solution.

In this example, the coupling strength $c$ is set to be zero. $10^{3}$ sample paths are computed with initial state $\mathbf{x}_{i}=(2.54+$ $\left.u_{i 1}, 2.52+u_{i 2}, 17.27+u_{i 3}\right)$, where $u_{i j}$ are randomly chosen from

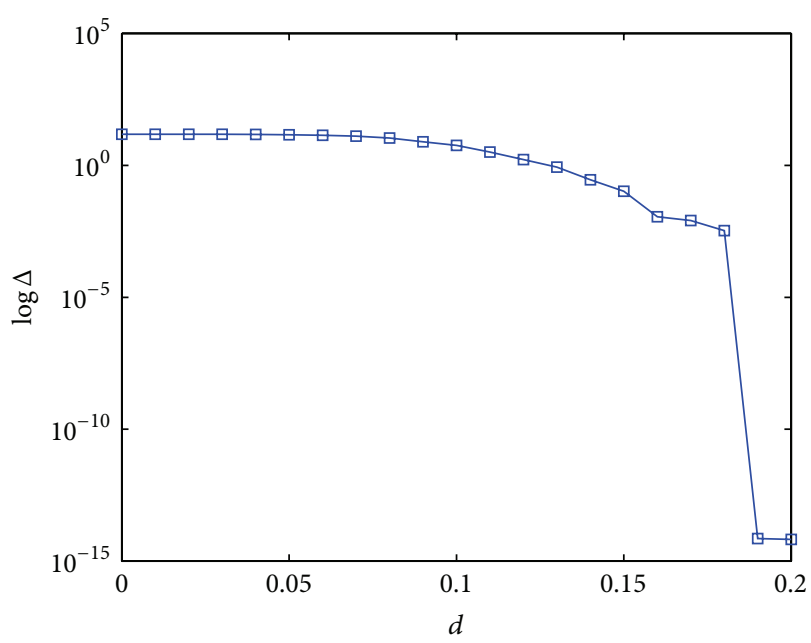

Figure 1: The evolution of $\Delta$ with respect to $d$ in three coupled Lorenz systems, when $c=0, T_{0}=4 \times 10^{3}$, and $T_{1}=5 \times 10^{3}$.

$[0,0.3]$. In Figure 1, we plot the evolution of $\Delta$ with respect to $d$. Form this figure, one can see that the scale $\Delta$ decreases with $d$ increasing, and three Lorenz systems just coupled by internal noise can achieve synchronization when the noise strength is large enough.

Example 2 (Rössler like system). The Rössler like system is dominated by

$$
\dot{\mathbf{x}}=\mathbf{D} \mathbf{x}+\mathbf{g}(\mathbf{x})
$$

where $\mathbf{x}=\left(x_{1}, x_{2}, x_{3}\right)^{T} \in R^{3}$ is the state vector, and

$$
\begin{gathered}
\mathbf{D}=\alpha\left(\begin{array}{ccc}
-\Gamma & -\beta & -\lambda \\
1 & \gamma & 0 \\
0 & 0 & -\eta
\end{array}\right), \quad f(x)= \begin{cases}0, & x<\theta \\
\zeta(x-\theta), & x \geq \theta\end{cases} \\
\mathbf{g}(\mathbf{x})=\left(\begin{array}{lll}
0 & 0 & \alpha \eta f\left(x_{1}\right)
\end{array}\right)^{T} .
\end{gathered}
$$

This system is a modified version of original Rössler system and has been used to study the synchronization of coupled chaotic systems in experiment. Under parameters $\alpha=1.0$, $\beta=1.5, \gamma=0.2, \eta=1.5, \Gamma=0.075, \lambda=0.75, \zeta=18.43$, and $\theta=0.5$, the system (21) has a chaotic attractor.

In this example, we set coupling strength $c=0.045$, and $10^{3}$ sample paths are computed with initial states $\mathbf{x}_{i}=$ $\left(-0.33+u_{i 1},-0.03+u_{i 2}, 0.01+u_{i 3}\right)$, where $u_{i j}$ are randomly chosen from [0,0.3]. In Figure 2, we plot the evolution of $\Delta$ with respect to $d$. From this figure, one can see that $\Delta$ is order of $10^{-2}$ when noise intensity $d=0$. With increase of $d$, the order of $\Delta$ decrease form $10^{-2}$ to $10^{-11}$. This demonstrates that multiplicative noise really plays a positive role in the synchronization of three coupled Rössler like systems. 


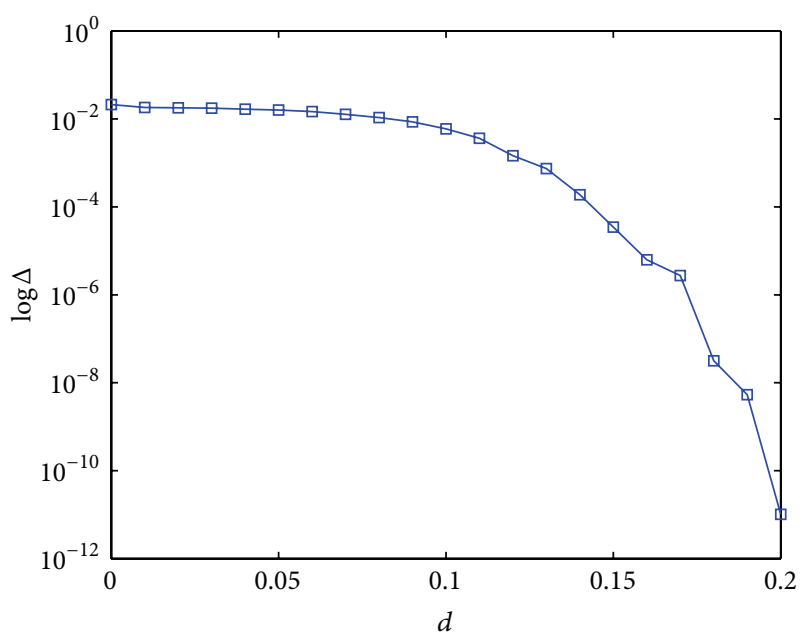

Figure 2: The evolution of $\Delta$ with respect to $d$ in three coupled Rössler like systems, when $c=0.045, T_{0}=4 \times 10^{3}$, and $T_{1}=5 \times 10^{3}$.

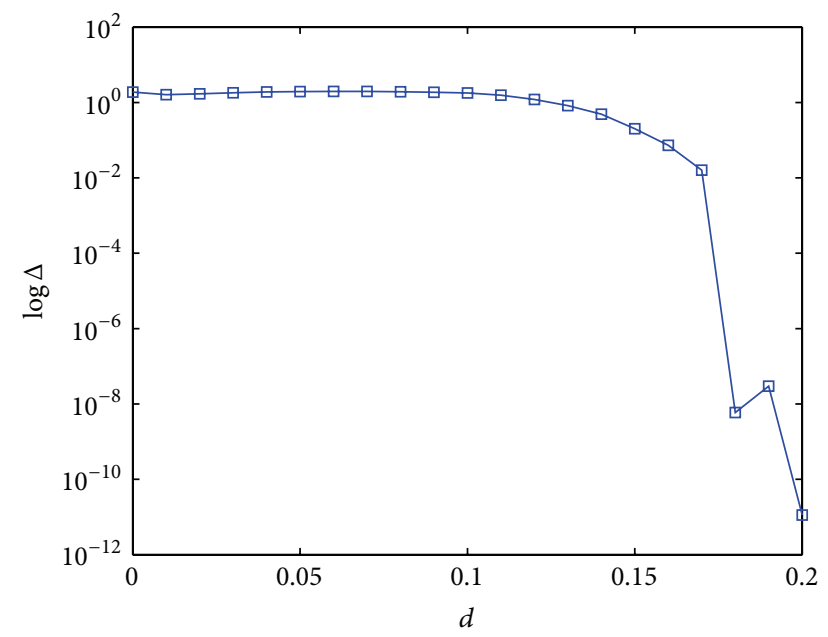

Figure 3: The evolution of $\Delta$ with respect to $d$ in three coupled Lorenz systems, when $c=0.002, T_{0}=4 \times 10^{3}$, and $T_{1}=5 \times 10^{3}$.

Example 3 (Hindmarsh-Rose (HR) neuron model). The HR neuron model can be described as follows:

$$
\begin{aligned}
& \dot{x}=y-a x^{3}+b x^{2}-z+I, \\
& \dot{y}=c-d x^{2}-y, \\
& \dot{z}=r(s(x-\chi)-z),
\end{aligned}
$$

where $x$ is the membrane potential and $y, z$ describe the transport of ions across the membrane through the ion channels. In this example, let the parameters $a=1, b=3$, $c=1, d=5, s=4, r=0.015, \chi=-1.6$, and external forcing $I=2.95$. Under above parameters, the system (23) exhibits chaotic spike.

In this example, we set coupling strength $c=0.002 .10^{3}$ sample paths are computed with initial states $\mathbf{x}_{i}=(1.05+$ $\left.u_{i 1},-4.71+u_{i 2}, 3.26+u_{i 3}\right)$ where $u_{i j}$ are randomly chosen from $[0,0.1]$. The evolution of $\Delta$ with respect to $d$ is plotted in
Figure 3. Form this figure, one can see that $\Delta$ is order of $10^{0}$ when noise intensity $d=0$. With increase of $d$, the order of $\Delta$ decreases to $10^{-11}$. This demonstrates that noise really plays a positive role in the synchronization of coupled HR neuron systems.

\section{Conclusions and Discussions}

To summarize, we study the role of multiplicative noise in the synchronization of unidirectionally coupled ring with three nodes. Based on the stability theory of stochastic differential equations, we demonstrate that multiplicative noise really has positive effect on synchronization. To demonstrate the correctness of the theoretical result, the Lorenz system, HR neuron system, and Rössler like system are employed as numerical examples. In this paper, we just study the coupled ring with three nodes and common noise. To be more practical, different nodes should be subjected to different noise. Moreover, many real systems should be described by networks with many nodes and complex structures. The role of multidimensional noise in the synchronization of complex dynamical network is one of our further works. For unidirectionally coupled ring with more than three nodes, we can not demonstrate that noise plays a positive role in synchronization by present method. We performed some numerical simulations for ring with many nodes. We found that noise plays a positive role in the synchronization of some samples, but some samples become unbounded. Is this unboundedness raised by artifact of finite precision of numerical simulation or determined by the structure of node system and ring? The theoretical result of this paper is acquired under the assumption of boundedness of ring. Though the bounded condition of the ring is difficult to be acquired by theoretical method, the bounded condition of network (2) is a problem which is worthwhile to be investigated. Further, the circuit experiment [50] is also best way to demonstrate the theoretical results, but the design of circuit is beyond the extent of our knowledge.

\section{Conflict of Interests}

The authors declare that there is no conflict of interests regarding the publication of this paper.

\section{Acknowledgments}

The authors are grateful for the support of the Special Foundation for Basic Scientific Research of Central Colleges, Foundation for Basic Scientific Research of Chang'an University (CHD2011JC006, CHD2013G2121017), and the National Natural Science Foundation of China (11202035, 11272258, 11201038, 61202437, and 11001031).

\section{References}

[1] A. Pikovsky, M. Rosenblum, and J. Kurths, Synchronization: A Universal Concept in Nonlinear Sciences, Cambridge unverisity press, Cambridge, Mass, USA, 2001. 
[2] S. Boccaletti, J. Kurths, G. Osipov, D. L. Valladares, and C. S. Zhou, "The synchronization of chaotic systems," Physics Report, vol. 366, no. 1-2, pp. 1-101, 2002.

[3] H. Fujisaka and T. Yamada, "Stability theory of synchronized motion in coupled-oscillator systems," Progress of Theoretical Physics, vol. 69, no. 1, pp. 32-47, 1983.

[4] L. M. Pecora and T. L. Carroll, "Synchronization in chaotic systems," Physical Review Letters, vol. 64, no. 8, pp. 821-824, 1990.

[5] L. M. Pecora and T. L. Carroll, "Driving systems with chaotic signals," Physical Review A, vol. 44, no. 4, pp. 2374-2383, 1991.

[6] M. G. Rosenblum, A. S. Pikovsky, and J. Kurths, "Phase synchronization of chaotic oscillators," Physical Review Letters, vol. 76, no. 11, pp. 1804-1807, 1996.

[7] M. G. Rosenblum, A. S. Pikovsky, and J. Kurths, "From phase to lag synchronization in coupled chaotic oscillators," Physical Review Letters, vol. 78, no. 22, pp. 4193-4196, 1997.

[8] Y. Sun and J. Cao, "Adaptive lag synchronization of unknown chaotic delayed neural networks with noise perturbation," Physics Letters A, vol. 364, pp. 277-285, 2007.

[9] L. Kocarev and U. Parlitz, "Generalized synchronization, predictability, and equivalence of unidirectionally coupled dynamical systems," Physical Review Letters, vol. 76, no. 11, pp. 18161819, 1996.

[10] X. Huang and J. Cao, "Generalized synchronization for delayed chaotic neural networks: a novel coupling scheme," Nonlinearity, vol. 19, no. 12, pp. 2797-2811, 2006.

[11] C. Li, X. Liao, and K. Wong, "Chaotic lag synchronization of coupled time-delayed systems and its applications in secure communication," Physica D: Nonlinear Phenomena, vol. 194, no. 3-4, pp. 187-202, 2004.

[12] L. Zhang, X. An, and J. Zhang, "A new chaos synchronization scheme and its application to secure communications," Nonlinear Dynamics, vol. 73, no. 1-2, pp. 705-722, 2013.

[13] M. A. Matías, V. Pérez-Muñuzuri, M. N. Lorenzo, I. P. Mariño, and V. Pérez-Villar, "Observation of a fast rotating wave in rings of coupled chaotic oscillators," Physical Review Letters, vol. 78, no. 2, p. 219, 1997.

[14] M. A. Matías and J. Güémez, "Transient periodic rotating waves and fast propagation of synchronization in linear arrays of chaotic systems," Physical Review Letters, vol. 81, p. 4124, 1998.

[15] Y. Kuramoto, Chemical Oscillations, Waves and Turbulence, Springer, Berlin, Germany, 1984.

[16] L. O. Chua, M. Itah, L. Kosarev, and K. Eckert, "Chaos synchronization in Chua's circuit," Journal of Circuits, Systems and Computers, vol. 3, no. 1, p. 93, 1993.

[17] H. N. Agiza and M. T. Yassen, "Synchronization of Rossler and Chen chaotic dynamical systems using active control," Physics Letters A, vol. 278, no. 4, pp. 191-197, 2001.

[18] D. Huang, "Simple adaptive-feedback controller for identical chaos synchronization," Physical Review E, vol. 71, Article ID 037203, 2005.

[19] B. Zhang and H. Li, "A new four-dimensional autonomous hyperchaotic system and the synchronization of different chaotic systems by using fast terminal sliding mode control," Mathematical Problems in Engineering, vol. 2013, Article ID 179428, 8 pages, 2013.

[20] X. Li, J. Gu, and W. Xu, "Adaptive integral observer-based synchronization for chaotic systems with unknown parameters and disturbances," Journal of Applied Mathematics, vol. 2013, Article ID 501421, 8 pages, 2013.
[21] A. S. Pikovsky, "Synchronization and stochastization of the ensemble of autogenrators by external noise," Radiophysics and Quantum Electronics, vol. 27, p. 576, 1984.

[22] R. V. Jensen, "Synchronization of randomly driven nonlinear oscillators," Physical Review E, vol. 58, Article ID R6907, 1998.

[23] A. Maritan and J. R. Banavar, "Chaos, noise, and synchronization," Physical Review Letters, vol. 72, no. 10, article 1451, 1994.

[24] A. S. Pikovsky, "Comment on "Chaos, noise, and synchronization"'” Physical Review Letters, vol. 73, no. 21, p. 2931, 1994.

[25] H. Herzel and J. Freund, "Chaos, noise, and synchronization reconsidered," Physical Review E, vol. 52, no. 3, pp. 3238-3241, 1995.

[26] G. Malescio, "Noise and synchronization in chaotic systems," Physical Review E, vol. 53, p. 6551, 1996.

[27] E. Sánchez, M. A. Matías, and V. Pérez-Muñuzuri, "Analysis of synchronization of chaotic systems by noise: an experimental study," Physical Review E, vol. 56, pp. 4068-4071, 1997.

[28] C. Lai and C. Zhou, "Synchronization of chaotic maps by symmetric common noise," Europhysics Letters, vol. 43, no. 4, article 376, 1998.

[29] R. Toral, C. R. Mirasso, E. Hernández-García, and O. Piro, "Analytical and numerical studies of noise-induced synchronization of chaotic systems," Chaos, vol. 11, no. 3, pp. 665-673, 2001.

[30] Y. Wu, J. Xu, D. He, and D. J. D. Earn, "Generalized synchronization induced by noise and parameter mismatching in Hindmarsh-Rose neurons," Chaos, Solitons and Fractals, vol. 23, no. 5, pp. 1605-1611, 2005.

[31] C. Zhou and J. Kurths, "Noise-induced synchronization and coherence resonance of a Hodgkin-Huxley model of thermally sensitive neurons," Chaos, vol. 13, no. 1, pp. 401-409, 2003.

[32] C. Zhou, J. Kurths, E. Allaria, S. Boccaletti, R. Meucci, and F. T. Arecchi, "Noise-enhanced synchronization of homoclinic chaos in a $\mathrm{CO}_{2}$ laser," Physical Review E, vol. 67, Article ID 015205(R), 2003.

[33] P. Tass, M. G. Rosenblum, J. Weule et al., "Detection of n:m phase locking from noisy data: application to magnetoencephalography," Physical Review Letters, vol. 81, no. 15, pp. 32913294, 1998.

[34] Z. Sun and X. Yang, "Generating and enhancing lag synchronization of chaotic systems by white noise," Chaos, vol. 21, Article ID 033114, 2011.

[35] W. Lin and Y. He, "Complete synchronization of the noiseperturbed Chua's circuits," Chaos, vol. 15, no. 2, Article ID 023705, 2005.

[36] W. Lin, "Realization of synchronization in time-delayed systems with stochastic perturbation," Journal of Physics. A. Mathematical and Theoretical, vol. 41, no. 23, 235101, 17 pages, 2008.

[37] A. Hu and Z. Xu, "Stochastic linear generalized synchronization of chaotic systems via robust control," Physics Letters A, vol. 372, no. 21, pp. 3814-3818, 2008.

[38] J. Cao, Z. Wang, and Y. Sun, "Synchronization in an array of linearly stochastically coupled networks with time delays," Physica A: Statistical Mechanics and its Applications, vol. 385, no. 2, pp. 718-728, 2007.

[39] W. Lin and G. Chen, "Using white noise to enhance synchronization of coupled chaotic systems," Chaos, vol. 16, no. 1, Article ID 013134, 2006.

[40] Y. Xiao, W. Xu, X. Li, and S. Tang, "The effect of noise on the complete synchronization of two bidirectionally coupled piecewise linear chaotic systems," Chaos, vol. 19, no. 1, Article ID 013131, 2009. 
[41] Y. M. Vega, M. Vázquez-Prada, and A. F. Pacheco, "Fitness for synchronization of network motifs," Physica A, vol. 343, no. 1-4, pp. 279-287, 2004.

[42] W. Horsthemke and R. Lefever, Noise-Induced Transitions: Theory and Application in Physics, Chemistry and Biology, Springer, Berlin, Germany, 1989.

[43] G. Pesce, A. McDaniel, S. Hottovy, J. Wehr, and G. Volpe, "Stratonovich-to-Itô transition in noisy systems with multiplicative feedback," Nature Communications, vol. 4, Article ID 2733, 2013.

[44] W. Lu and T. Chen, "New approach to synchronization analysis of linearly coupled ordinary differential systems," Physica D. Nonlinear Phenomena, vol. 213, no. 2, pp. 214-230, 2006.

[45] L. Arnold, Stochastic Differential Equation and Application, Academic, New York, NY, USA, 1972.

[46] R. C. Hilborn, Chaos and Nonlinear Dynamics, Oxford university press, Oxford, UK, 1994.

[47] J. L. Hindmarsh and R. M. Rose, "A model of neuronal bursting using three coupled first order differential equations," Proceedings of the Royal Society of London B, vol. 221, no. 1222, pp. 87-102, 1984.

[48] I. A. Heisler, T. Braun, Y. Zhang, G. Hu, and H. A. Cerdeira, "Experimental investigation of partial synchronization in coupled chaotic oscillators," Chaos, vol. 13, no. 1, pp. 185-194, 2003.

[49] P. E. Kloeden and E. Platen, Numerical Solution of Stochastic Differential Equations, Springer, Berlin, Germany, 1995.

[50] B. Nana and P. Woafo, "Synchronization in a ring of four mutually coupled van der Pol oscillators: theory and experiment," Physical Review E, vol. 74, no. 4, Article ID 046213, 2006. 


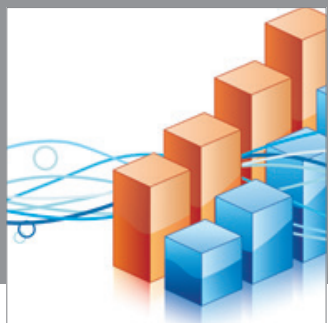

Advances in

Operations Research

mansans

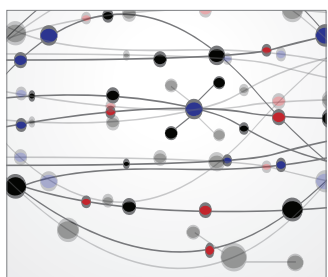

The Scientific World Journal
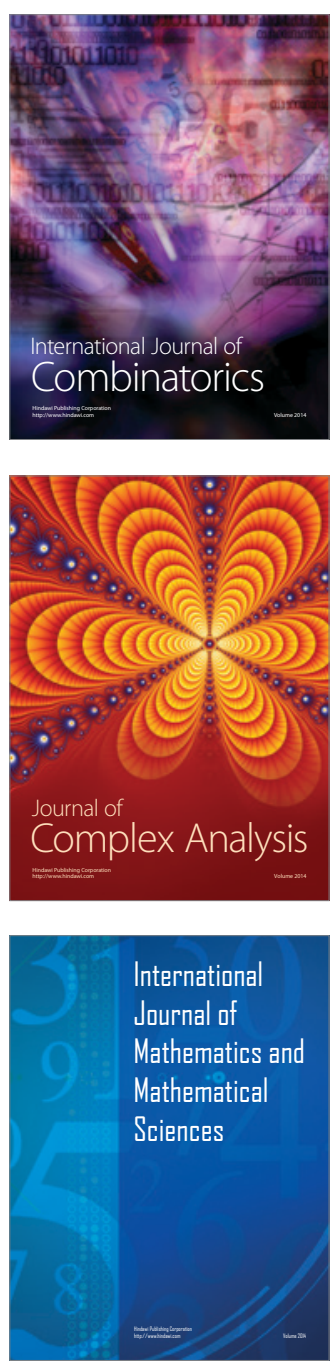
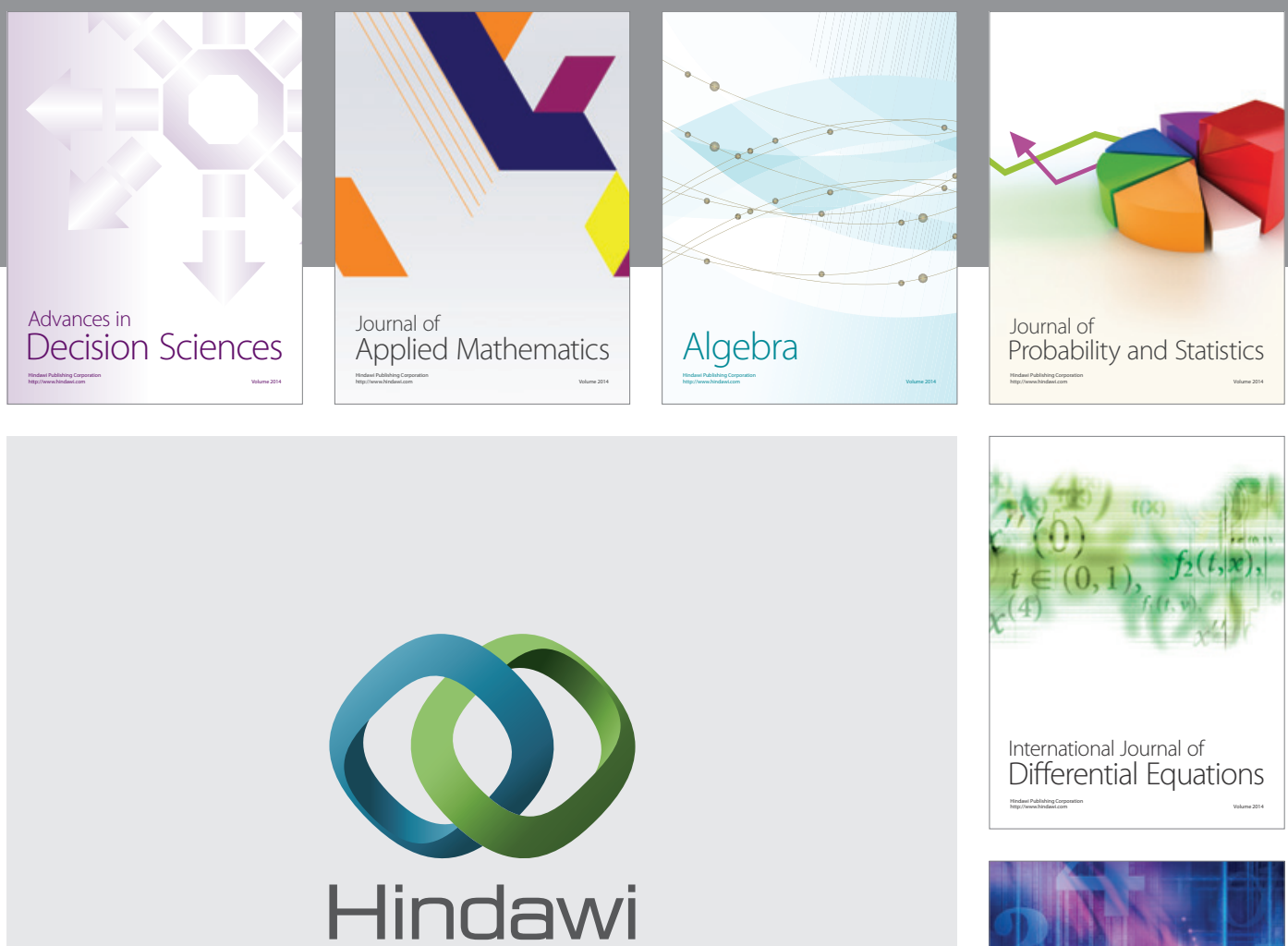

Submit your manuscripts at http://www.hindawi.com
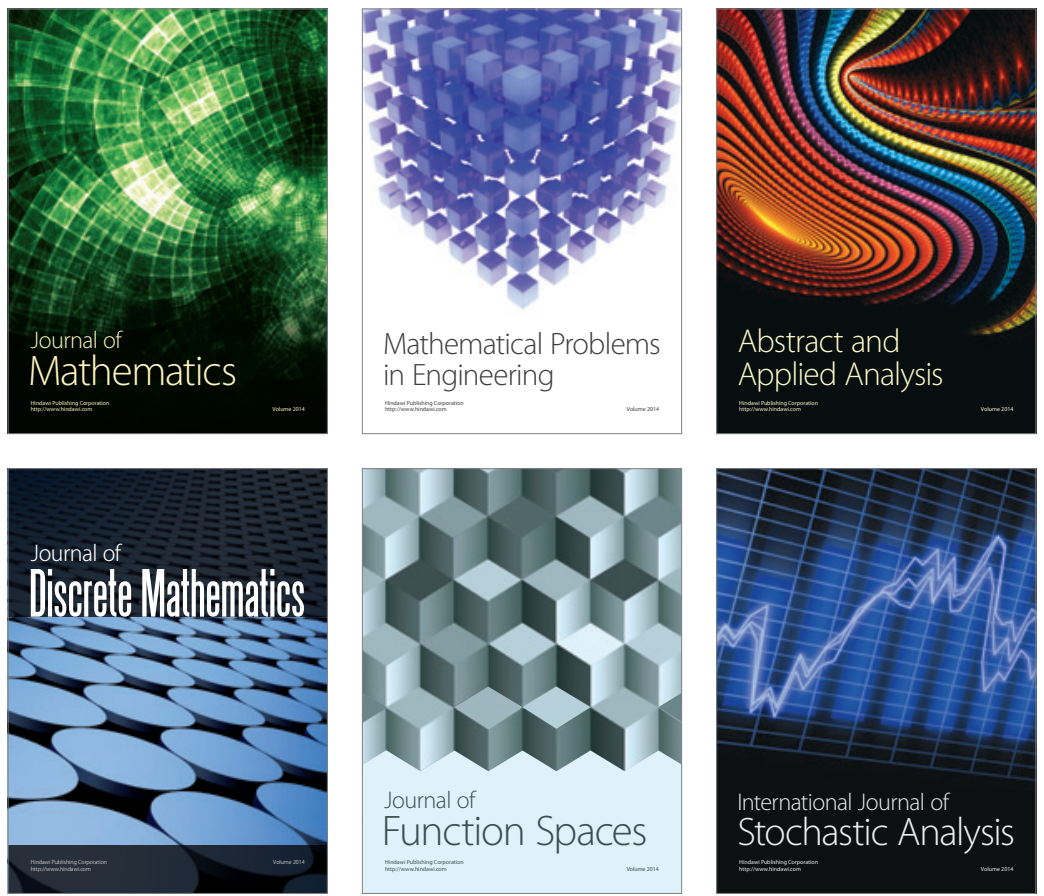

Journal of

Function Spaces

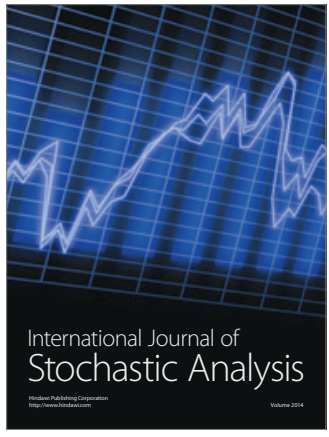

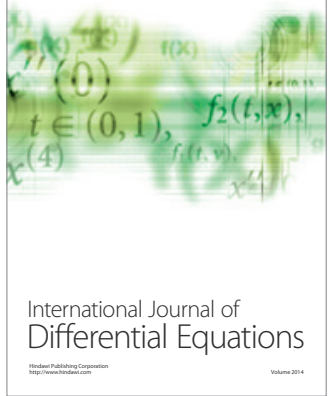
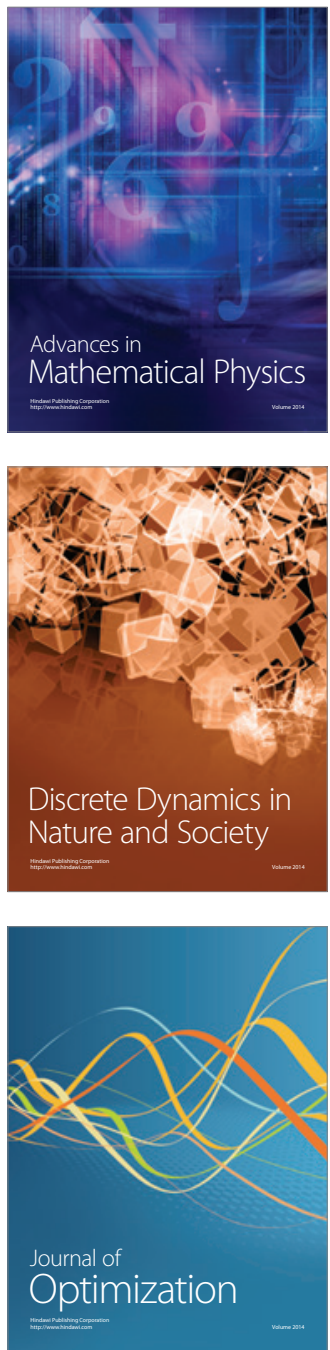